\title{
CFD simulation and experiment on the flow field of air-assisted ultra-low-volume sprayers in facilities
}

\author{
Xinyu Lu, Yan Gong*, Dejiang Liu, Guo Wang, Xiao Chen, Xiao Zhang, Qiuhan Liang \\ (Nanjing Institute for Agricultural Mechanization, Ministry of Agriculture and Rural Affairs, Nanjing 210014, China)
}

\begin{abstract}
In order to explore the performance of the B-ULV-616A knapsack sprayer, computational fluid dynamics (CFD) was used to simulate the B-ULV-616A knapsack air-assisted device, which features an ultra-low-volume electric sprayer. Field experiments were carried out to test the spraying effects, and the KANOMAX anemometer was used to verify the simulated results. First, the internal and external flow fields and droplet deposition distribution of the ultra-low-volume sprayer were established. The results showed that the air-assisted spray device can change the airflow speed and direction and produce a high-speed swirling airflow at the outlet of the air-assisted spray device. The high-speed airflow (maximum of $83.5 \mathrm{~m} / \mathrm{s}$ ) generates negative pressure (minimum of $0.099 \mathrm{MPa}$ ) and causes a rapid increase in the droplet velocity and a secondary droplets spray, allowing droplets to reach a longer distance. Then, the maximum relative error was $20.14 \%$, and its average value was $9.59 \%$, indicating that the CFD method is suitable for the flow field analysis of the air-assisted spray device. Finally, based on the greenhouse experiment, the knapsack air-assisted ultra-low-volume electric sprayer was found to effectively improve the deposition on the rear of the crop, increase the droplet density (maximum of $81 / \mathrm{cm}^{2}$; droplet density of conventional electric sprayer is $64 / \mathrm{cm}^{2}$ ), and reduce the deposition amount and coefficient of variation (below 20\%) within and between regions. Further, it managed to reduce pesticide use (by 69.85\%) and rural non-point source pollution.
\end{abstract}

Keywords: CFD simulation, air-assisted sprayer, ultra-low spraying rate, deposition, air-velocity field, relative error DOI: $10.25165 /$ j.jjabe.20211402.6013

Citation: Lu X Y, Gong Y, Liu D J, Wang G, Chen X, Zhang X, et al. CFD simulation and experiment on the flow field of air-assisted ultra-low-volume sprayers in facilities. Int J Agric \& Biol Eng, 2021; 14(2): 26-34.

\section{Introduction ${ }^{1}$}

According to statistical data, the annual rate of crop yield in facilities in China has decreased by $20 \%$ to $30 \%$ owing to disease and pesticides ${ }^{[1]}$. Currently, the main disease prevention measure remains pesticide spraying on the plant canopy. However, the knapsack manual (electric) sprayer or stretcher (cart) power sprayer currently used in facility greenhouses have a high formulation rate, poor spray uniformity, and low pesticide efficacy. Moreover, the sudden humidity increase in facility greenhouses caused by mass spraying may make plants extremely susceptible to secondary diseases $^{[2]}$. In the middle and late growth stages of crops, such as watermelons, melons, and tomatoes, mutual occlusion between crops occurs owing to the high density of the crop canopy. It is

Received date: 2020-07-16 Accepted date: 2021-01-03

Biographies: Xinyu Lu, MS, Assistant Researcher, research interests: equipment for crop protection and pesticide application technology, Email: luxinyu1996@163.com; Dejiang Liu, MS, Assistant Researcher, research interests: equipment for crop protection and pesticide application technology, Email: liudejiang@caas.cn; Guo Wang, MS, Assistant Researcher, research interests: equipment for crop protection and pesticide application technology, Email: 512829304@qq.com; Xiao Chen, MS, Assistant Researcher, research, interests: equipment for crop protection and pesticide application technology, Email: 1205787558@qq.com; Xiao Zhang, MS, Assistant Researcher, research interests: equipment for crop protection and pesticide application technology, Email: zhangxiao_xhx@126.com; Qiuhan Liang, MS, Assistant Researcher, research interests: equipment for crop protection and pesticide application technology, Email: liangqiuhan@qq.com.

*Corresponding author: Yan Gong, Professor, research interests: equipment for crop protection and pesticide application technology. Nanjing Institute of Agricultural Mechanization, Ministry of Agriculture and Rural Affairs, Nanjing 210014, China. Tel: +86-25-84346241, Email: jsgymm@ hotmail.com. difficult for traditional spraying equipment to reach the inner crop canopy. Therefore, plant protection in facilities requires novel equipment with better spray uniformity, lower formulation rate, and stronger droplet penetrability.

Air-assisted spray technology deploys a high-speed fan-generated airflow to drive the droplet movement ${ }^{[3]}$, thus reaching target crops at longer distances and driving blade-disturbance. Good coverage of different application distances and crop types can be achieved by adjusting the spray parameters. Consequently, air-assisted spraying has been widely used in orchard plant protection. In recent years, scholars have applied computational fluid dynamics (CFD) to the study of air-assisted spraying ${ }^{[3-17]}$. Dekeyser et al. ${ }^{[18]}$ used CFD simulation and experimental verification methods to study the parameters of nozzles, droplet size distribution, and wind field airflow on three commercially available air-assisted sprayers with different air-feeding devices. Hong et al. ${ }^{[19]}$ combined crop canopy deposition with wind-driven sprayers and studied the droplet movement and drift using the Lagrangian model. Duga et al. ${ }^{[21]}$ conducted CFD simulations and field test research on four different crop cultivation models using three types of air-assisted spray equipment. The test results prove that strong wind can effectively improve crop target deposition. Garcerá et al. ${ }^{[20]}$ conducted an application study on citrus trees using an axial-flow air blower sprayer and a pneumatic sprayer. Then, they compared the application results of the standard cone and Venturi nozzles and proved that the airflow can effectively reduce droplet drift and deposition losses. Qi et al. ${ }^{[22]}$ established the droplet deposition distribution model of the HardiLB-255 orchard air-assisted sprayer, while Wang et al. ${ }^{[23]}$ determined the time required by the droplets to attach to the target crop surface. Further, Sun et al. ${ }^{[24]}$ deployed 
CFD technology to study the characteristics of droplet deposition and particle size distribution under different wind speed, spray height, and pressure conditions and experimentally verified the results. $\mathrm{Li}$ et al. ${ }^{[25]}$ studied the droplet deposition characteristics of air-assisted spraying on citrus and proved that increasing the angle of air delivery led to a slight increase in the simulation results and experimental errors. Moreover, the feasibility of using CFD technology to study the wind field airflow and deposit rate of the air-assisted spray equipment was verified. However, the abovementioned studies are mostly related to orchard air-assisted spray equipment, and the research objective is mostly the study of the external flow field of the air-assisted spray device. Little research has been conducted on the internal flow field of spraying machinery and air distribution devices for facility greenhouses.

In this study, CFD technology was deployed to study the internal and external flow field distribution and particle movement trajectory of an air-assisted spray device. The model and grid were built using the Creo Parametric and ICEM CFD software, and the Fluent software was used to establish the flow and spray field model of the air-assisted spray device. Then, the simulation results were verified through laboratory tests and the simulation errors were determined. Moreover, a greenhouse spray test was conducted to determine the field operation effect of the knapsack air-assisted ultra-low-spraying-rate electric sprayer. The results provide a reference for the further research and development of new spray components in precision spraying equipment and their efficient application in pest-control greenhouse crops.

\section{Materials and methods}

\subsection{Sprayer}

The test sprayer is the B-ULV-616A knapsack air-assisted ultra-low-spraying-rate electric sprayer (hereafter, referred to as ultra-low-spraying-rate sprayer) produced by Shenzhen Longray Technology Co., Ltd.; its structure is shown in Figure 1. The ultra-low-spraying-rate sprayer is composed of a base (battery, power switch, indicator light, and control system), fan, air-assisted-spray device (medicine liquid adjustment knob, air speed switch, infusion tube, air delivery telescopic tube, and air-assisted spray nozzle), and spray tank. Unlike the electric knapsack sprayer designed by Wang et al. ${ }^{[29]}$ (a micro diaphragm pump was used to pressurize and transport the chemical liquid to the spray head, while the axial flow fan only provided wind power), this ultra-low-spraying-rate sprayer generates high-pressure air only through the fan to the spray tank to pressurize its spray mixture and through the infusion tube to the nozzle to atomize the liquid. These measures prevent usage of a liquid pump, reduce the weight of the machine while providing certain pressure, and are easily adjustable.

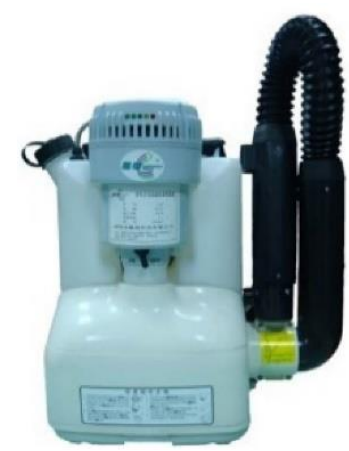

Figure 1 B-ULV-616A knapsack air-assisted ultra-low spraying rate electric sprayer
The key component of the ultra-low-spraying-rate sprayer is the air-assisted spray device. Its working principle is that the battery drives the fan to generate a high-speed rotary cutting airflow. Part of the airflow enters the spray tank to pressurize the spray mixture and transport it to the air-assisted spray device through the nozzle bar. The other part of the airflow is sent to the air-assisting spray device through the telescopic air duct, and the direction and speed of the airflow are changed by the vortex air deflector of the core component in the air-assisting spray device. The pressurized airflow merges with the droplet sprayed from the air-assisted spray device, and then performs a secondary spraying causing the droplet to move along the axis of the air-assisted spray device. The fan speed and spraying rate can be adjusted by the air speed switch and spray mixture adjustment knob, respectively.

\subsection{CFD simulation}

\subsubsection{Build geometric model}

The air-assisted spray device, which is the key component of the ultra-low-spraying-rate sprayer, cannot only increase the air velocity but also change the direction of the airflow. The vortex air deflector, which is the core component of the air-assisted spray device, is irregularly shaped and greatly influences the flow of the airflow fields. The presence of internal and external flow fields is necessary. The Creo Parametric 4.0 M050 software (PTC Corporation, USA) installed on an HP Z840 computer (Intel(R) Xeon(R) CPU E5-2650 v3@2.30GHz processor) was used to build the geometric model of the air-assisted spray device, as shown in Figure 2. The geometric parameters of the air-assisted spray device are as follows. The external size parameter of the air cylinder is $\varphi 56 \mathrm{~mm} \times 74 \mathrm{~mm}$, the internal size parameter is $\varphi 49 \mathrm{~mm} \times 69 \mathrm{~mm}$, and the blade height of the vortex air deflector is $26 \mathrm{~mm}$. The device comprises a total of four blades, which can change the direction of the airflow in the clockwise direction. At the center of the vortex air deflector there is a nozzle (the center of end face $\mathrm{C}$ is the spray hole) with a tip diameter of $3 \mathrm{~mm}$. The vortex air deflector and air cylinder are connected by screws.

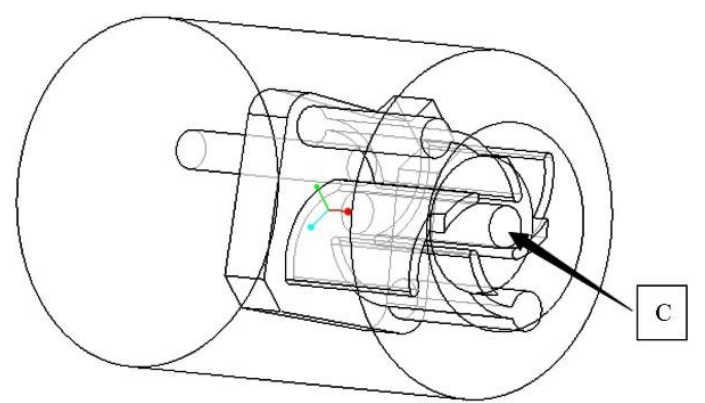

Figure 2 Geometric model of the air-assisted spray device

The CFD model simulates the entire fluid domain. Before the simulation, a Boolean operation created using the Creo Parametric software was performed to obtain the fluid domain of the air-assisted spray device. Currently, the typical values of the height and width of the flow field simulation of the air assisted device $^{[16]}$ are 3-4 times the size of the air duct; herein, values 5 times the size of the air duct are used. The length is determined based on practical application of the air-supplied spray device and simplification of the simulation calculations. The distance between the machine and crop during application is approximately $500 \mathrm{~mm}$. Therefore, lengths of $500 \mathrm{~mm}$ and $2000 \mathrm{~mm}$ were tested in the simulations. Through the comparison of the results, the length of $1000 \mathrm{~mm}$ was found to be most appropriate. Finally, an external flow field with a size of $500 \mathrm{~mm} \times 500 \mathrm{~mm} \times 1000 \mathrm{~mm}$ was set at the air-assisted spray device outlet, as shown in Figure 3. 


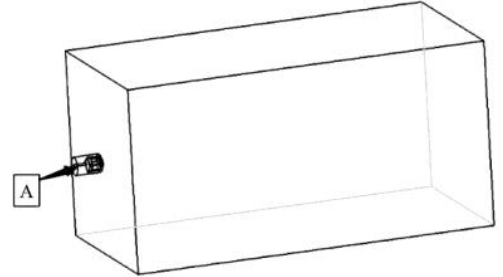

Figure 3 Flow field of air-assisted spray device

\subsubsection{Meshing}

ICEM CFD 20 (ANSYS, Inc., PA, USA) is a professional meshing software, which can achieve functions such as meshing, pre-processing, and boundary condition setting, and is widely used in the field of CFD mesh generation. The fluid model developed in Creo Parametric was saved in STEP format and imported into the ICEM 20.0 software for meshing. Owing to the irregular internal shape of the air-assisted spray device and the existence of rounded corners, arcs, and other complex structures, the Tetra/Mixed mesh type was selected. Following grid division, 15804906 grid units and 2816854 nodes were generated. After inspection, the grid meets the calculation requirements; the grid model is shown in Figure 4.

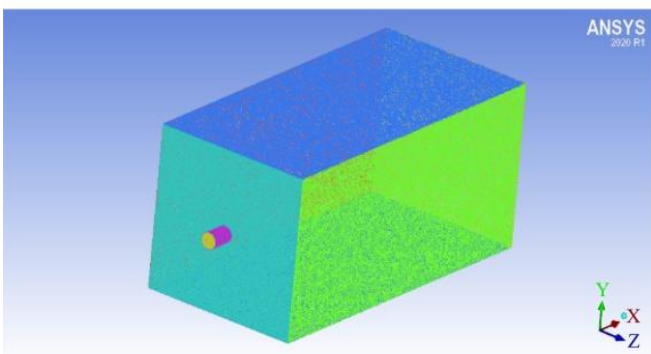

Figure 4 Grid model

\subsubsection{Selection of fluid calculation mode}

According to Qi et al. ${ }^{[22]}$, spraying is a discrete fluid motion in a continuous fluid, which belongs to the air-liquid two-phase flow motion of the multiphase flow. As the discrete phase corresponds to the droplets and the continuous phase corresponds to air, the volume ratio is very low (below 10\%). The droplets only exist in the form of deposition, evaporation, and drift in the movement of the external flow field. The discrete phase exchanges momentum, mass, and energy with the continuous phase during movement. Owing to the rotation effect around the location of the vortex air deflector in the continuous phase and the high air velocity near the outlet of the air-assisted spray device, a realizable $k-\varepsilon$ model (Equations (1) and (2)) with turbulence correction was used in the continuous phase flow model.

Turbulent kinetic energy $(k)$ equation:

$$
\begin{array}{r}
\frac{\partial}{\partial_{t}}(\rho k)+\frac{\partial}{\partial_{x_{j}}}\left(\rho k \mu_{j}\right)=\frac{\partial}{\partial_{x_{j}}}\left[\left(\mu+\frac{\mu_{t}}{\sigma_{k}}\right) \frac{\partial}{\partial_{x_{j}}}\right]+ \\
G_{k}+G_{b}-\rho \varepsilon-Y_{M}+S_{k}
\end{array}
$$

Turbulent energy dissipation rate $(\varepsilon)$ equation:

$$
\begin{array}{r}
\frac{\partial}{\partial_{t}}(\rho \varepsilon)+\frac{\partial}{\partial_{x_{j}}}\left(\rho \varepsilon \mu_{j}\right)=\frac{\partial}{\partial_{x_{j}}}\left[\left(\mu+\frac{\mu_{t}}{\sigma_{\varepsilon}}\right) \frac{\partial \varepsilon}{\partial_{x_{j}}}\right]+ \\
\rho C_{1} S_{\varepsilon}-\rho C_{2} \frac{\varepsilon^{2}}{k+\sqrt{v \varepsilon}}+C_{1 \varepsilon} \frac{\varepsilon}{k} C_{3 \varepsilon} G_{b}+S_{\varepsilon}
\end{array}
$$

where, $\quad C_{1}=\max \left[0.43, \frac{\eta}{\eta+5}\right], \eta=S \frac{k}{\varepsilon}, S=\sqrt{2 S_{i j} S_{i j}} \cdot \rho$ is fluid density, $\mathrm{kg} / \mathrm{m}^{3} ; t$ is time, $\mathrm{s} ; x_{j}=1,2,3$ (1-3 represent the $X, Y Z$ directions, respectively); $\mu_{t}$ is eddy viscosity, $\mu_{j}$ is air velocity, $\mathrm{m} / \mathrm{s} ; k$ is turbulence kinetic energy, $\mathrm{J} ; \varepsilon$ is dissipation rate; $G_{k}$ represents the generation of turbulence kinetic energy due to the mean velocity gradients; $G_{b}$ is the generation of turbulence kinetic energy due to buoyancy; $Y_{m}$ represents the contribution of the fluctuating dilatation in compressible turbulence to the overall dissipation rate; $C_{l}, C_{2}$ and $C_{1 \varepsilon}$ are constants; $C_{3 \varepsilon}$ is a coefficient related to buoyancy in the calculation of the flow of compressible fluids, when the main flow direction is parallel to the direction of gravity, $C_{3 \varepsilon}=1$, and when it is vertical, $C_{3 \varepsilon}=0 ; \sigma_{k}$ and $\sigma_{\varepsilon}$ are the turbulent Prandtl numbers for $k$ and $\varepsilon$, respectively; $S_{k}$ and $S_{\varepsilon}$ are user-defined source terms. Other parameters refer to the ANSYS Help 20.0. Finally, a Lagrangian discrete phase model (DPM) was used to simulate the actual droplet motion.

$$
\begin{gathered}
\frac{\partial \alpha_{q}}{\partial t}+\bar{u} \cdot a_{q}=\frac{S_{a_{q}}}{\rho_{q}} \\
\rho=\alpha_{q} \cdot \rho_{q}+\left(1-\alpha_{q}\right) \rho_{q} \\
\frac{\partial}{\partial_{t}}(\rho \cdot \bar{u})+\nabla(\rho \cdot \bar{u} \cdot \bar{u})=\nabla p+\left[\mu\left(\nabla \bar{u}+\nabla \bar{u}^{T}\right)\right]+\rho \cdot \bar{g}+\bar{F}
\end{gathered}
$$

where, $\alpha_{q}$ is the volume ratio of the $q$-th phase flow; $S_{a_{q}}$ is the generalized source term; $\rho$ is the density, $\mathrm{kg} / \mathrm{m}^{3} ; \rho_{q}$ is the density of the $q$-th phase, $\mathrm{kg} / \mathrm{m}^{3} ; g$ is the acceleration due to gravity, $\mathrm{m} / \mathrm{s}^{2}$; $\nabla$ is the gradient operator; $p$ is the pressure, $\mathrm{Pa} ; F$ is the volume force term, $\mathrm{N}$.

\subsubsection{Simulation parameters setting}

The continuous phase material was considered to be air and was treated as a fluid of a constant temperature of $25^{\circ} \mathrm{C}$. The velocity at the inlet of the air-assisted spray device was set to $5.7 \mathrm{~m} / \mathrm{s}$ based on laboratory measurements, and the Reynolds number $\left(R e \approx 2.48 \times 10^{4}>2300\right)$ was calculated using Equation (4), which describes the turbulent state. Therefore, the realizable $k-\varepsilon$ turbulence model was used to simulate the continuous phase, whereas the velocity-inlet was used to simulate the flow field inlet. The turbulence intensity $I=4.5 \%$ was calculated from Equation (5), and the hydraulic diameter was set to $45 \mathrm{~mm}$. The pressure-outlet was used as the boundary condition for the flow field outlet, and the pressure was set to $0.1013 \mathrm{MPa}$. As shown in Figure 3, the internal and external flow fields were calculated as a whole flow field; the surface, A, of the air-assisted spray device was the velocity-inlet; and the top, front, left, back, and right boundary in the external flow field area were pressure-outlets; the rest of the boundaries were considered to be walls.

$$
R e=\frac{V L}{v}
$$

where, $V$ is cross-sectional average velocity, $\mathrm{m} / \mathrm{s} ; L$ is the characteristic length, $\mathrm{m} ; v$ is kinetic viscosity of the fluid, $\mathrm{mm}^{2} / \mathrm{s}$.

$$
I=0.16 \cdot \operatorname{Re}^{-0.125}
$$

The discrete phase material was considered to be water with a density, droplet surface tension, and viscosity of $998.2 \mathrm{~kg} / \mathrm{m}^{3}$, $0.072 \mathrm{~N} / \mathrm{m}$, and $0.001 \mathrm{~kg} / \mathrm{m} \cdot \mathrm{s}$, respectively. The type of the discrete phase injection is the surface, and the released from surfaces is surface C. Given the influence of wind on atomization, droplet deformation and fragmentation were considered in the DPM, so a Taylor analogy breakup model was selected. The bottom and right boundary conditions of the external flow field were considered to be wall-jet, and the remaining inlet, outlet, and wall boundary conditions were set to escape or trap. The total flow rate of the droplets was $0.005 \mathrm{~kg} / \mathrm{s}$, and the droplet velocity was $0.18 \mathrm{~m} / \mathrm{s}$. The droplet size in the calculation follows the Rosin-Rammler distribution, i.e., the droplet diameter is the actual measurement value of the Bettersize 2000S Laser particle size analyzer in the laboratory. Therefore, the minimum, maximum, and mean droplet diameter was $26 \mu \mathrm{m}, 98 \mu \mathrm{m}$, and $56 \mu \mathrm{m}$, respectively. Finally, the spread parameter, $n=1.36$, was calculated from Equation (6), the number of particles was 100, and the start and stop times were $0 \mathrm{~s}$ and $1 \mathrm{~s}$, respectively. 


$$
Y_{d}=e^{-(d / \bar{d})^{n}}
$$

where, $d$ is the droplet diameter, $\mu \mathrm{m} ; n$ is the spread parameter; $\bar{d}$ is the mean droplet diameter, $\mu \mathrm{m}$; and $Y_{d}=e^{-1} \approx 0.368$.

The control solver uses a pressure-based solver to perform transient calculations with a $0.01 \mathrm{~s}$ time step, time size of 100 , and scaled residuals of 0.001 .

\subsection{Design of simulation experiment of airflow velocity field}

In order to determine the accuracy of the airflow velocity simulation in Fluent, a simulation result verification test was carried out in the laboratory of the Inspection and Testing Center of Nanjing Institute of Agricultural Mechanization, Ministry of Agriculture and Rural Affairs of China. The test conditions were the same as the simulation conditions. A KANOMAX anemometer was used to measure the velocity values at different distances in the axial direction of the air-assisted spray device in the simulation area of Fluent. A total of 10 sample points were arranged every $0.1 \mathrm{~m}$ from the air-assisted spray device. During testing, the air-assisted spray device was fixed on the bracket and placed horizontally. The angle of the wind cylinder was $0^{\circ}$. The KANOMAX meteorological anemometer was used to measure the wind speed at each measurement point. The measurements were repeated three times at each measurement point, and the average was recorded.

\subsection{Field experiment}

\subsubsection{Test site and equipment}

In order to test the actual field droplet size of the ultra-low-volume electric sprayer and the field test effect, a field spray test was carried out. The experiment was conducted on May 15, 2019 in a vegetable greenhouse in the Jiangsu Hua'an Modern Agricultural Industrial Park, Huai'an District, Huai'an City, Jiangsu Province of China. The room temperature was $35.45^{\circ} \mathrm{C}$ and the humidity was $55.39 \%$. The selected hanging vine watermelon, and the test equipment were the ultra-low-sprayingrate sprayer and knapsack electric sprayer (CK).

2.4.2 Sampling method and arrangement

Figure 5 is a schematic diagram of the sample points considered during testing. Three repeats were arranged along the walking direction, and three sample points were arranged per repeat. Each sample point was divided into three layers: upper, middle, and lower (Figure 6). A filter paper was placed on the front of the leaves, and a water-sensitive paper was placed on both the front and back leaves of the crop. Each layer of filter paper and water-sensitive paper had to be kept uncovered in the cloth sample. In the test, the ALLURA RED solution was used as a tracer and was added to clean water at a concentration (mass ratio) of $0.5 \%$. The water-sensitive paper and filter paper were recovered immediately after spraying and were completely dried. A scanner was deployed to scan and save the water-sensitive paper, and the filter paper was stored in a sealed bag. Then, the droplet analysis software DepositScan (developed by the US Department of Agriculture) was used to perform image processing on the water-sensitive paper (Figure 7) to analyze the droplet density.

The filter paper sample was fully immersed in a specified amount of water until the Allura red dye (Zhejiang Dragoni Colour Technology Co., Ltd., China) on the paper was completely precipitated. The absorbance value of the elution solution was measured via a visible light spectrophotometer and converted into the concentration value of the red dye in the elution solution according to the conversion formula; the relationship between the concentration value of the Allura red solution and the corresponding absorbance value was determined in advance by a spectrophotometer. The deposition amount per unit area of the lure red tracer on each sampling filter paper was calculated using Equation (7).

$$
p=\frac{f(A) \times V}{S}=\frac{f(A) \times V}{\pi r^{2}}
$$

where, $p$ is the amount of drug solution deposited per unit area, $\mu \mathrm{g} / \mathrm{cm}^{2} ; A$ is the absorbance value of the washed out Allura red solution; $f(A)$ is the concentration of Allura red dye in the washed-out Allura red solution, $\mu \mathrm{g} / \mathrm{mL} ; V$ is the amount of fresh water added when soaking the filter paper sample, $\mathrm{mL} ; s$ is the area of the disc filter paper, $\mathrm{cm}^{2}$; and $r$ is the radius of the disc filter paper, $\mathrm{cm}$.

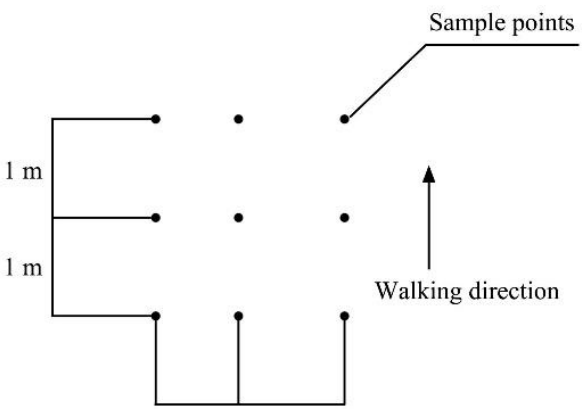

Figure 5 Schematic diagram of the sample points

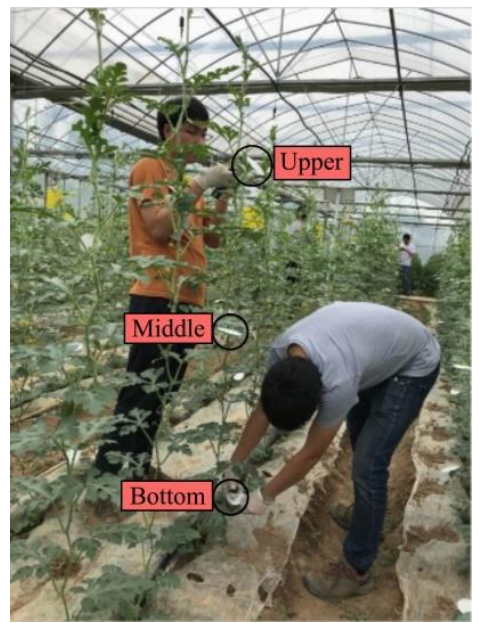

Figure 6 Schematic layout

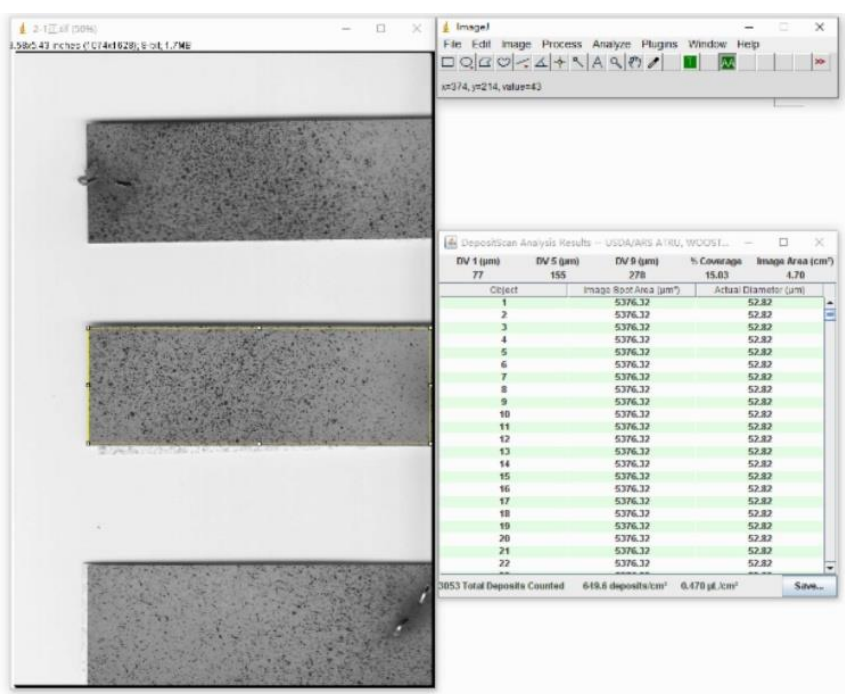

Figure 7 Processing interface of the droplet analysis software Deposit Scan

The spraying test was carried out in the selected test area using the ultra-low-spraying-rate and knapsack electric sprayers. 
During testing, the test machine was adjusted to the rated condition and spray according to the actual application situation in the field. The experiment was repeated three times, and the average values were recorded. After testing, the spray working area was measured, and the dosage per unit area was calculated according to Equation (8).

$$
q=\frac{G-G_{1}}{S}
$$

where, $Q$ is the pesticide application dose rate, $\mathrm{L} / \mathrm{hm}^{2} ; G$ is the volume of spray mixture in the tank, $\mathrm{L} ; G_{1}$ is the volume of rest spray mixture in the tank, L; and $S$ is the application area, $\mathrm{hm}^{2}$.

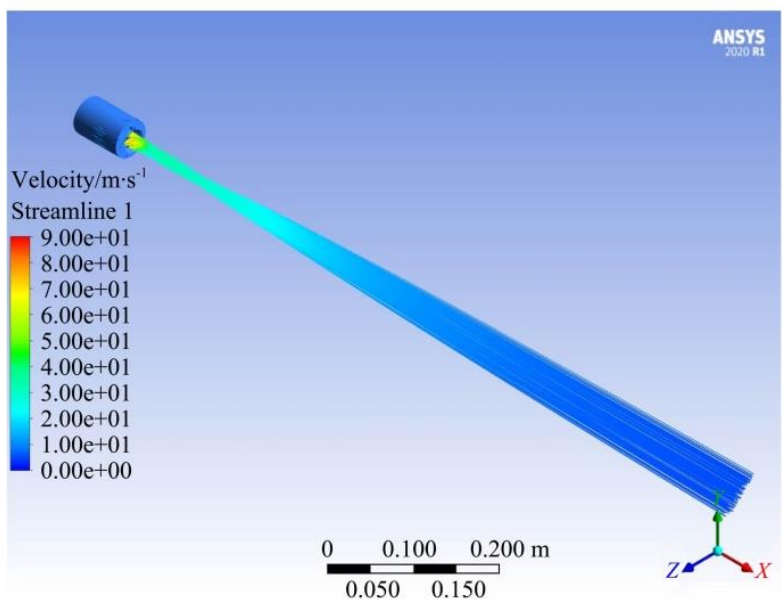

a. Isometric Projection

Figure 8 Streamlines

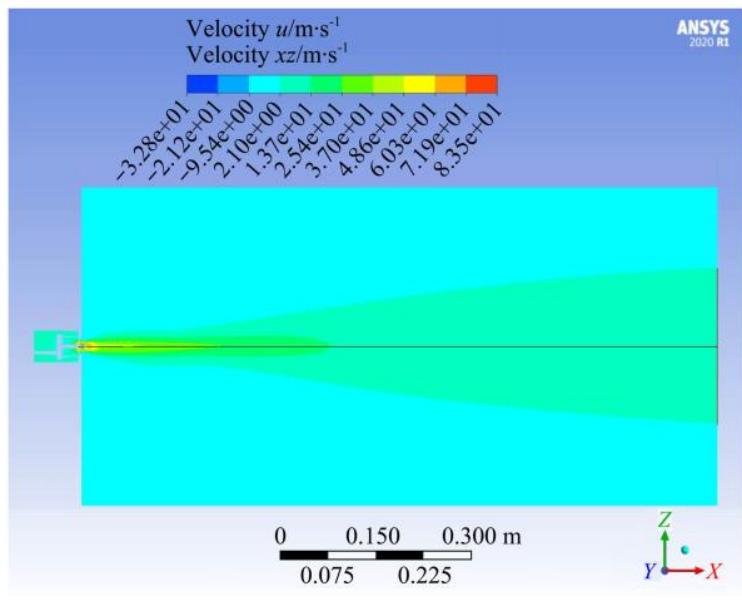

a. $X-Z$ plane

Figure 9 Velocity contours in different planes

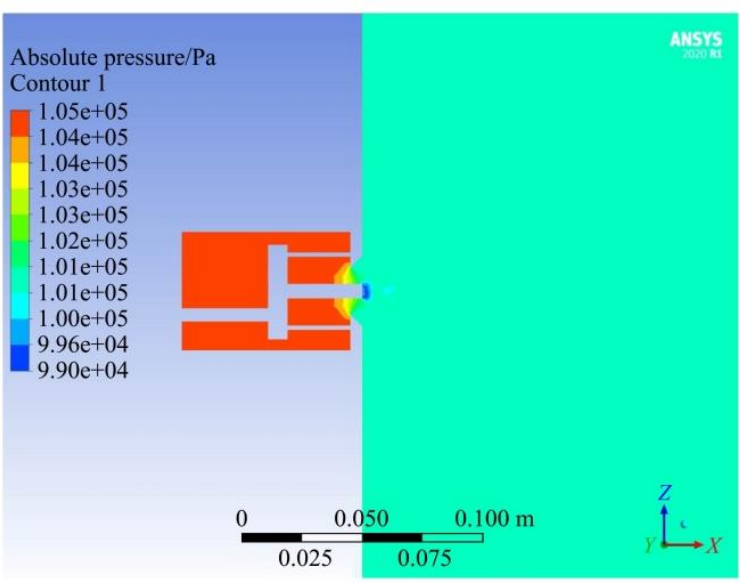

a. $X-Y$ plane

\section{Results and analysis}

\subsection{Simulation results}

The Fluent calculation data were imported into the CFD-POST 2020 R1 software for processing. The CFD-POST software can display the data in a scientific way, which improves comprehension and facilitates the discovery of physical characteristics. The airflow streamlines are shown in Figure 8, the velocity contours in the $Y=0$ and $Z=0$ planes are illustrated in Figures $9 a$ and $9 b$, the pressure cloud diagram is plotted in Figures 10a and 10b, and the droplet distribution is shown in Figure 11.

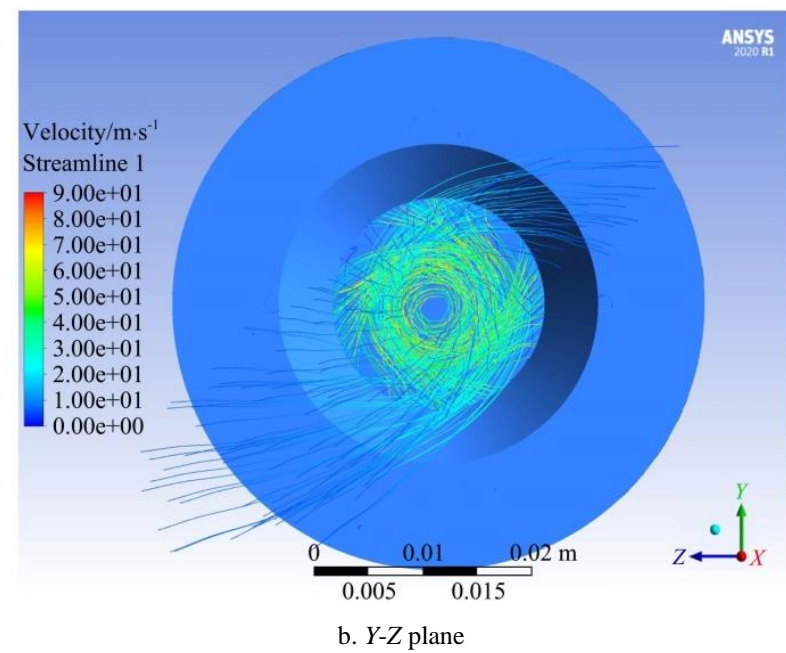

b. $Y-Z$ plane

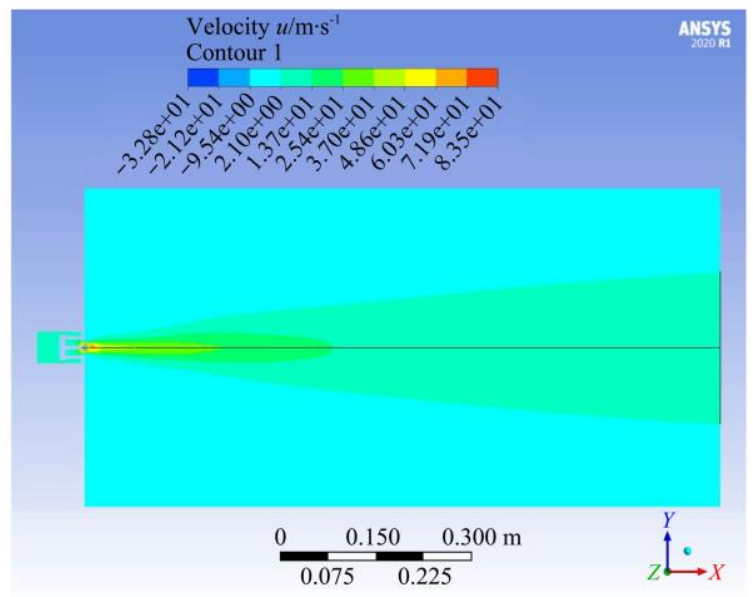

b. $X-Y$ plane

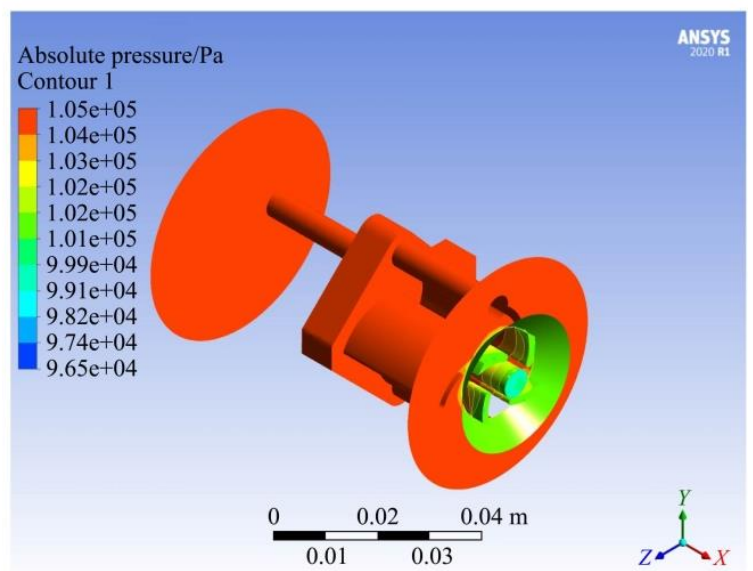

b. Isometric Projection

Figure 10 Pressure contours near the outlet of the device 


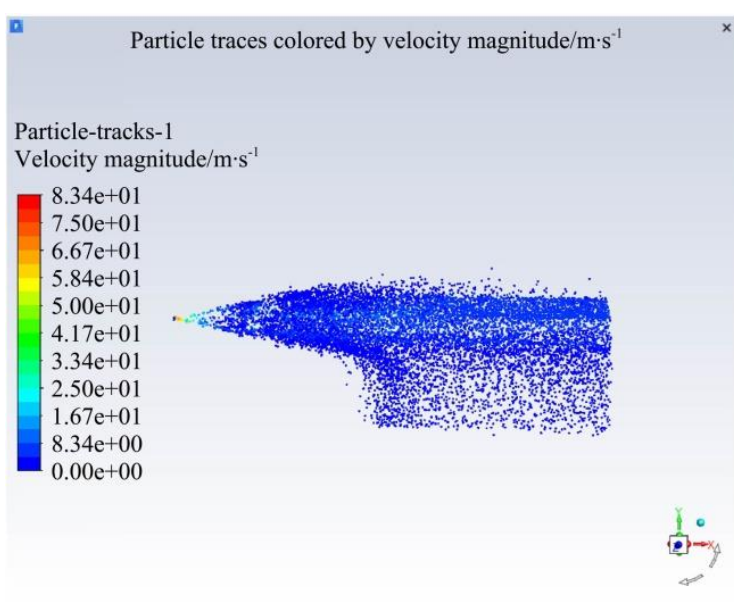

a. $X-Y$ plane

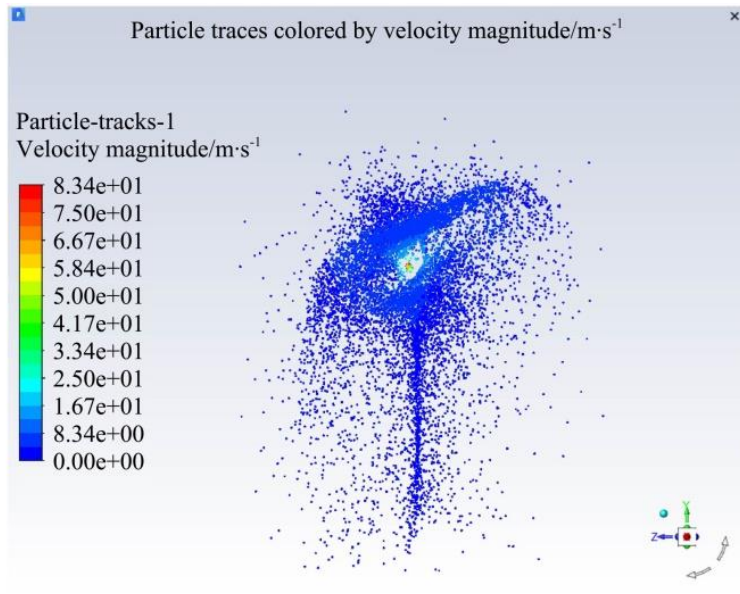

b. $Y$-Z plane

Figure 11 Droplets distribution at different planes

\subsubsection{Path lines distribution}

Figure 8a indicates that the airflow enters the air-assisted spray device from surface $A$. When passing through the vortex air deflector, the airflow is blocked by the air cylinder; thus, its direction is changed and its speed is increased, generating a high-speed airflow at the outlet of the air-assisted spray device (near surface $C$ ). Following, a rotary cut airflow enters the outer flow field moving along the axis of the air-assisted spray device $(X$ positive direction) accompanied by radial diffusion and circumferential rotation. Owing to the small area at the outlet of the airflow and the large space in the outer flow field, the airflow has a rotating effect when entering the outer flow field. Meanwhile, the airflow speed decreases rapidly owing to air resistance, and the speed streamline gradually diverges. Near surface $\mathrm{C}$, the airflow speed is high, the rotation is strong, and the streamlines are concentrated. According to Figures $8 \mathrm{a}$ and $8 \mathrm{~b}$, after early rotation, the rotation effect of the airflow streamline is weakened, the overall streamline tends to move linearly, and the distribution of the streamline on the right side of the right boundary becomes spindle-shaped.

\subsubsection{Velocity contours}

The velocity contours in Figures $9 \mathrm{a}$ and $9 \mathrm{~b}$ show that, in the flow field of the air-assisted spray device, the airflow speed is significantly increased owing to the air cylinder, and the speed at the outlet of the air-assisted spray device in the inner flow field reaches a maximum of $83.5 \mathrm{~m} / \mathrm{s}$. In the area of the external flow field, the airflow velocity gradually decreases with the increase of the moving distance, and attenuation becomes more evident. The airflow velocity on the air-assisted spray device corresponds to the highest value of the airflow on this cross section. In the $Y=0$ and $Z=0$ planes, the velocity distribution of the external flow field of the distance air-assisted spray device is almost symmetric along the axis, and the velocity on both sides of the axis is basically equal. Additionally, the airflow velocity equivalent area increases along the air-assisted spray device, and the airflow diffuses along the duct radial. The wind speed at the inlet is only $5.7 \mathrm{~m} / \mathrm{s}$, while the wind speed at the outlet is approximately 15 times that at the inlet. This may be because the cross-sectional area ratio of the inlet and outlet of the air-assisted spray device is approximately 10.47, and its relation to changes in the velocity and area conforms to the Bernoulli equation; thus, it exhibits a high-speed increase and pressure change. Because the sectional area at the outlet of the air-assisted spray device is small and the area of the external flow field is large, the airflow attenuation after entering the external flow field is strong. The internal structure of the air-assisted spray device is complex, and the airflow velocity and direction as well as change in these parameters at different positions differ when entering the outer drainage basin. Simultaneously, the airflow is subject to air resistance and gravity during movement, which results in a decrease in the airflow velocity.

\subsubsection{Pressure distribution}

The pressure contours in Figure 10a indicate that, when the airflow enters the air-assisted spray device, the air cylinder increases not only the airflow velocity but also the internal pressure of the air-assisted spray device. The pressure value on the left side of the outlet of the air-assisted spray device reaches the maximum value of $0.105 \mathrm{MPa}$. Figure 10 shows that the airflow velocity at this position reaches the maximum value. After entering the external flow field area, the air pressure outlet of the flow field suddenly drops and generates a negative pressure; following, it rises back to the atmospheric pressure at a position approximately $0.1 \mathrm{~m}$ from the outlet of the air-assisted spray device. According to Bernoulli's equation ${ }^{[26]}$, in a constant-height flow, where the velocity is high, the pressure is small. Therefore, when the airflow enters the outer flow field through the outlet of the air-assisted spray device, the pressure drops at the location of maximum flow velocity, generating a negative pressure area (near surface C). This occurs owing to the high airflow speed and pressure difference between the inner and outer flow fields. The pressure in the negative pressure area is less than the pressure of the tank, which is conducive to the transportation of the spray mixture from the spray tank to the spray nozzle and the secondary spray of the mist droplets from the spray nozzle. From Figure 10b, it can be found that the pressure at the outlet of the air-assisted spray device changes significantly (from a maximum of $1.05 \mathrm{MPa}$ to a minimum of $0.099 \mathrm{MPa}$ ), whereas the pressure at other positions remains almost unchanged. During the simulation, the pressure of the air-assisted spray device is changed.

\subsubsection{Spray distribution}

Figure 11a presents the distribution of the droplets after they are sprayed by the nozzle (surface $\mathrm{C}$ ). The droplet velocity rapidly increases along the axial direction and diverges in the circumferential direction of the assisted spray device. Then, the droplet speed decreases sharply and diverges forward in a conical shape. At approximately $0.5 \mathrm{~m}$ from the nozzle, the number of droplets on the upper side decreases, and the droplets on the bottom side gradually increase. Through analysis of the droplet movement duration, we find that some low-velocity droplets 
gradually begin to deposit. At $0.5-1.0 \mathrm{~m}$, the droplets on the bottom boundary deposition gradually increase; part of the high-velocity droplets escape towards the right boundary at $1 \mathrm{~m}$ away from the nozzle. This is attributed to the rapid increase in the droplet velocity and the secondary droplet production. However, owing to the particularity of the air-assisted spray device, the droplet speed decreases with the attenuation of the airflow, so the droplets exhibit conical divergence. During movement, the droplets are combined via the airflow, air, and gravity. Some droplets with relatively low velocity or large mass deposit gradually, whereas high-velocity droplets reach the right boundary with the airflow and escape.

\subsection{Experimental verification}

\subsubsection{Comparison of test value and simulation value}

To determine the accuracy of the airflow velocity simulation using Fluent, a model verification test was conducted. The relative error method to measure the accuracy of the simulation and results is given by Equation (9).

$$
E=\frac{\left|V_{s}-V_{a}\right|}{V_{a}} \times 100 \%
$$

where, $V_{s}$ is the Fluent simulation value; $V_{a}$ is the laboratory test value; $E$ is the relative error.

Table 1 shows that the value obtained via Fluent to simulate the airflow velocity field of the air-assisted spray device is basically consistent with the test value. The relative error of multiple measurements is less than $20.14 \%$, the relative mean deviation is $9.59 \%$, and the area with an error exceeding $15 \%$ is concentrated within $0.5-1.0 \mathrm{~m}$ around the simulated area. This is owing to the error between the simulated value and the test value caused by the simplified model. In the simulation process, the temperature change of the airflow was not considered. However, during the test process, there was a certain temperature difference between the temperature of the outlet of the air-assisted spray device and the environment, which has been ignored in the simulation experiment. Simultaneously, the wind speed value at each point during the laboratory test was not fixed but fluctuated within a certain range, and the final value was considered to be the average of multiple measurements. Overall, the feasibility of the Fluent simulation was demonstrated; the average error is small, and the simulation results are accurate.

Table 1 Test values, simulated values, and relative errors of the wind speed

\begin{tabular}{cccc}
\hline Distance $/ \mathrm{m}$ & Simulated value $/ \mathrm{m} \cdot \mathrm{s}^{-1}$ & Test value $/ \mathrm{m} \cdot \mathrm{s}^{-1}$ & Relative error $E / \%$ \\
\hline 0.1 & 35.70 & 37.17 & 4.11 \\
0.2 & 25.70 & 27.42 & 6.70 \\
0.3 & 20.50 & 19.28 & 5.93 \\
0.4 & 15.30 & 13.90 & 9.14 \\
0.5 & 13.30 & 10.71 & 19.46 \\
0.6 & 10.20 & 8.92 & 12.58 \\
0.7 & 8.40 & 7.66 & 8.82 \\
0.8 & 8.00 & 6.74 & 15.75 \\
0.9 & 7.50 & 6.05 & 19.31 \\
1 & 7.00 & 5.59 & 20.14 \\
\hline
\end{tabular}

\subsection{Facility greenhouse test}

3.3.1 Distribution uniformity of the droplet deposition

The distribution uniformity of the droplet deposition is one of the important parameters for evaluating the spraying effect of the spraying equipment ${ }^{[27]}$. Therefore, the uniformity of the deposition distribution of the ultra-low-spraying-rate and knapsack-type electric sprayers was studied. Table 2 shows the maximum and minimum deposition amounts of the ultra-lowspraying-rate sprayer are $2031.52 \mu \mathrm{g} / \mathrm{cm}^{2}$ and $1524.77 \mu \mathrm{g} / \mathrm{cm}^{2}$, respectively, and their difference is $506.75 \mu \mathrm{g} / \mathrm{cm}^{2}$. The coefficient of variation is less than $20 \%$, indicating high deposition distribution uniformity. The maximum and minimum deposition amounts of the knapsack-type electric sprayer are $8656.06 \mu \mathrm{g} / \mathrm{cm}^{2}$ and $2074.47 \mu \mathrm{g} / \mathrm{cm}^{2}$, respectively, and their difference is $6581.59 \mu \mathrm{g} / \mathrm{cm}^{2}$. The coefficient of variation within the same area reaches $54.72 \%$, and that between regions can be as high as $56.21 \%$, indicating poor deposition distribution uniformity. Overall, the deposition amount of the ultra-low-spraying-rate sprayer is lower than that of the knapsack-type electric sprayer; however, the ultra-low-spraying-rate sprayer exhibits higher deposition uniformity as the deposition amounts of the upper, middle, and bottom leaves of the canopy are comparable. The upper layer of the knapsack-type electric sprayer has the highest deposition amount, and the bottom layer has a bottom droplet distribution.

Table 2 Deposition distribution of different pesticide application equipment

\begin{tabular}{ccccc}
\hline & $\begin{array}{c}\text { Collection } \\
\text { area }\end{array}$ & $\begin{array}{c}\text { Deposit } \\
\text { rate } \\
/ \mu \mathrm{g} \cdot \mathrm{cm}^{-2}\end{array}$ & $\begin{array}{c}\text { Coefficient of } \\
\text { variation within the } \\
\text { same area } \mathrm{CV}_{1} / \%\end{array}$ & $\begin{array}{c}\text { Coefficient of } \\
\text { variation between } \\
\text { regions } \mathrm{CV}_{2} / \%\end{array}$ \\
\hline $\begin{array}{c}\text { Ultra-low } \\
\text { spraying rate } \\
\text { sprayer }\end{array}$ & upper & 2031.52 & 18.87 & 13.53 \\
\hline middle & 1524.77 & 17.59 & \\
Knapsack & bottom & 1561.22 & 17.41 & 56.21 \\
electric & upper & 8656.06 & 42.73 & \\
\hline sprayer & middle & 4010.55 & 40.75 & \\
\hline
\end{tabular}

\subsubsection{Deposition distribution}

According to Yuan and Wang ${ }^{[28]}$, when the droplet density per unit area reaches to a critical value, a good control effect can be produced. Pest control of watermelon crops is mainly concentrated on the back of the leaves, so it is necessary to evaluate the droplet density distribution on crop leaves, especially on the back of the leaves. Figure 12 indicates that the minimum droplet density of the ultra-low-spraying-rate sprayer is approximately $40 \mathrm{~cm}^{-2}$, and the highest is $81 \mathrm{~cm}^{-2}$. The droplet densities of the upper, middle, and bottom layers are similar, and the average value is approximately $80 \mathrm{~cm}^{-2}$ and $50 \mathrm{~cm}^{-2}$ on the front and back, respectively. The maximum droplet density of the knapsack-type electric sprayer is $65 \mathrm{~cm}^{-2}$, and the lowest is $7 \mathrm{~cm}^{-2}$. The droplet density on the back of the crop leaves is significantly lower than that on the front of the crop leaves. The spray distribution is not evenly distributed.

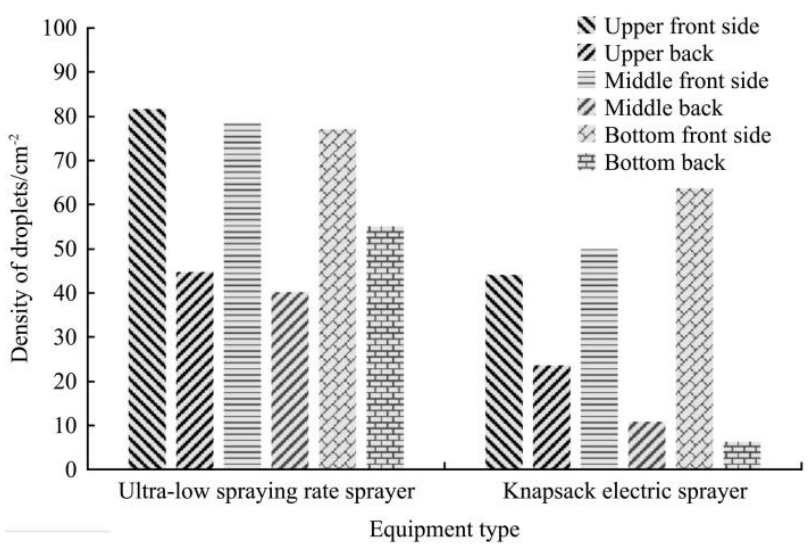

Figure 12 Droplet density distribution of different application equipment 


\subsubsection{Droplet size}

The DepositScan software was deployed to analyze the droplet size, $d_{s}$, on water-sensitive paper using Equation (10).

$$
d=0.95 d_{s}^{0.91}
$$

The droplet sizes of the upper front and back layers are $75.8 \mu \mathrm{m}$ and $76.65 \mu \mathrm{m}$, respectively; that of the middle front and back layers are $64.95 \mu \mathrm{m}$ and $37.54 \mu \mathrm{m}$, respectively; and that of the bottom front and back are $37.63 \mu \mathrm{m}$ and $46.28 \mu \mathrm{m}$, respectively. The droplet size data range for water-sensitive paper is within the droplet size range obtained via CFD simulation.

\subsubsection{Amount of pesticide application per unit area}

According to Equation (8), the application rate per unit area of the knapsack-type electric sprayer is $304.5 \mathrm{~L} / \mathrm{hm}^{2}$, whereas that of the ultra-low-spraying-rate sprayer is $91.8 \mathrm{~L} / \mathrm{hm}^{2}$; this corresponds to a $69.85 \%$ reduction of the application amount. The ultra-low-spraying-rate sprayer can also reduce the amount of crop deposition while obtaining higher droplet density and deposition distribution uniformity. Compared with the large-capacity knapsack-type electric sprayer featuring the rain-type spraying method, the ultra-low-spraying-rate sprayer can reduce the pesticide application amount while ensuring successful application, thus reducing agricultural chemical use and increasing efficiency.

\section{Conclusions}

In this study, CFD technology was used to study the internal and external flow fields and spray distribution of the air-assisted spray device of the ultra-low-spraying-rate sprayer. Combined with laboratory and field tests, the consistency of the CFD simulation results was verified, and the actual field effect of the sprayer was tested. The results are as follows:

1) The airflow generated by the fan of the ultra-low-sprayingrate sprayer increases the speed of the airflow when passing through the air-assisted spray device and changes the direction of the airflow when passing through the vortex air deflector. The airflow has a significant rotation component along the direction of the vortex air deflector and enters the outer flow flied from the outlet of the air-assisted spray rate device. The airflow speed at the outlet of the device reaches a maximum value of $83.5 \mathrm{~m} / \mathrm{s}$ and generates a negative pressure area. The pressurized spray mixture is delivered to the nozzle, sprayed through the airflow, and transported with the airflow. As the distance increases, the wind speed and the droplet speed gradually decrease. The droplets gradually deposit on the bottom boundary, and some of them escape from the spray field at the right boundary at $1 \mathrm{~m}$.

2) The relative error method was used to compare the simulated and measured values of the wind speed on the axis of the air-assisted spray device. The estimated relative error was $20.14 \%$, and the average value was $9.59 \%$. These values demonstrate the feasibility of CFD implementation for the simulation of the flow field of the air-assisted spray device.

3) The ultra-low-spraying-rate sprayer can increase the droplet density, improve the uniformity of the spray distribution, lower the coefficient of variation, and reduce the amount of pesticide applied per unit area, thereby achieving to limit the use of pesticides. The droplet size distribution on water-sensitive paper was found to be in good agreement with the droplet size value simulated via CFD. This further proves the feasibility of CFD simulation.

In this study, CFD simulation is not linked to the crop canopy, and does not consider the impact of the crop canopy on the wind field and spray deposition. In further research, the crop canopy, spray angle, and wind speed changes can be considered to determine the effect of the spray mixture.

\section{Acknowledgements}

This work was financially supported by the National Key Research and Development Program of China (Grant No. 2017YFD0200303), the National Key Research and Development Program of China (Grant No. 2018YFD0201304), and the China Agriculture Research System (Grant No. CARS-25).

\section{[References]}

[1] Liu D J, Gong Y, Wang G, Chen X, Zhang X, Wu G Z. Advances in mechanization of pesticide application technology equipment in facility agriculture. Journal of Agricultural Mechanization Research, 2017; 39(5): 6-11. (in Chinese)

[2] He X K. Changing the present situation of my country's plant protection machinery and pesticide application technology is seriously lagging behind Transactions of the CSAE, 2004; 1: 13-15. (in Chinese)

[3] Gu J B, Ding W M, Qiu W, Sun C D. Research status and trends of variable orchard application machinery and application technology in orchards. Journal of Fruit Science, 2014; 31(6): 1154-1157. (in Chinese)

[4] Musiu W M, Qi L, Wu Y. Evaluation of droplets size distribution and velocity pattern using Computational Fluid Dynamics modelling. Computers and Electronics in Agriculture, 2019; 164: 104886.

[5] Delele M A, Jaeken P, Debaer C, Baetens K, Endalew A M, Ramon H, et al. CFD prototyping of an air-assisted orchard sprayer aimed at drift reduction. Computers \& Electronics in Agriculture, 2007; 55(1): 16-27.

[6] Abramzon B, Sirignano W A. Droplet vaporization model for spray combustion calculations. International Journal of Heat and Mass Transfer, 1989; 32(9): 1605-1618.

[7] Bartzanas T, Kacira M, Zhu H, Karmakar S, Tamimi E, Katsoulas N, et al. Computational fluid dynamics applications to improve crop production systems. Computers and Electronics in Agriculture, 2013; 93: 151-167.

[8] Zhang B, Tang Q, Chen L P, Zhang R R, Xu M. Numerical simulation of spray drift and deposition from a crop spraying aircraft using a CFD approach. Biosystems Engineering, 2018; 166: 184-199.

[9] Hołownicki R, Doruchowski G, Świechowski W, Godyń A, Konopacki P J. Variable air assistance system for orchard sprayers; concept, design and preliminary testing. Biosystems Engineering, 2017; 163: 134-149.

[10] Endalew A M, Debaer C, Rutten N, Vercammen J, Delele M A, Ramon H, et al. Modelling pesticide flow and deposition from air-assisted orchard spraying in orchards: A new integrated CFD approach. Agricultural and Forest Meteorology, 2010; 150(10): 1383-1392.

[11] Song S R, Xia H B, Liu H S, Hong T S, Sun D Z, Lu Y H. Numerical simulation and experiment of the structure optimization of the air-blast sprayer nozzle. Transactions of the CSAM, 2013; 44(6): 73-78, 55. (in Chinese).

[12] Yuan X, Qi L J, Ji R H, Zhang J H, Wang H, Huang S K. Airflow velocity field and droplet deposition characteristics analysis of greenhouse air-supply mist eliminator. Transactions of the CSAM, 2012; 43(8): 7177. (in Chinese).

[13] Peng J. Numerical simulation of the flow field in the air-sending ultra-low volume spray device. MS dissertation. Wuhan: Wuhan University of Technology, 2006; 60p. (in Chinese).

[14] Xu Y M, Zhu X W, Liu Z J, Hu Y H, Gu F. Field simulation and structure optimization of the air conveying system in air assisted sprayer based on computer fluid dynamics. Journal of Zhejiang University (Agriculture and Life Science Edition), 2018; 44(4): 451-458. (in Chinese)

[15] Lü X L, Zhang M N, Chang Y H, Lei X H, Yang Q S. Influence of deflector angles for orchard air-assisted sprayer on 3D airflow distribution. Transactions of the CSAE, 2017; 33(15): 81-87. (in Chinese)

[16] Li M. Development and experimental research of 3M-50 autonomous walking greenhouse machine. Doctoral dissertation. Tai'an: Shandong Agricultural University, 2017. (in Chinese)

[17] Zhou L F, Xue X Y, Jia W D, Ding S M, Sun Z. Application and prospect analysis of CFD technology in wind spray of fruit trees. Journal of Drainage and Irrigation Machinery Engineering, 2014; 32(9): 776-782.

[18] Dekeyser D, Duga A T, Verboven P, Endalew A M, Hendrickx N, Nuyttens D. Assessment of orchard sprayers using laboratory experiments and computational fluid dynamics modelling. Biosystems 
Engineering, 2013; 114(2): 157-169.

[19] Hong S W, Zhao L, Zhu H. CFD simulation of pesticide spray from air-assisted sprayers in an apple orchard: Tree deposition and off-target losses. Atmospheric Environ, 2018; 175: 109-119.

[20] Garcerá C, Moltó E, Chueca P. Spray pesticide applications in Mediterranean citrus orchards: Canopy deposition and off-target losses. Science of the Total Environment, 2017; 599-600: 1344-1362.

[21] Duga A T, Ruysen K, Dekeyser D, Nuyttens D, Bylemans D, Nicolai B M, et al. Spray deposition profiles in pome fruit trees: Effects of sprayer design, training system and tree canopy characteristics. Crop Protection, 2015: 67: 200-213.

[22] Qi L J, Zhao Y Q, Wang J, Ji R H, Mang L. CFD simulation and experimental verification of droplet dispersion of air-assisted orchard sprayer. Transactions of the CSAM, 2010; 41(2): 62-67. (in Chinese)

[23] Wang J X, Qi L J, Xia Q J. CFD simulation and validation of trajectory and deposition behavior of droplets around target affected by air flow field in greenhouse. Transactions of the CSAE, 2015; 31(11): 46-53. (in
Chinese)

[24] Sun G X, Wang X C, Ding W M, Zhang Y. Simulation analysis of droplet deposition characteristics based on CFD discrete phase model. Transactions of the CSAE, 2012; 28(6): 13-19. (in Chinese)

[25] Li J, Zhao C Q, Li S J, Chen H, Ding S F. Droplet deposition characteristics of CFD based orchard air-driven sprayer. Journal of Huazhong Agricultural University, 2019; 38(6): 171-177. (in Chinese)

[26] Yan D G. The total flow Bernoulli equation in fluid mechanics. Physics and Engineering, 2014; 24(4): 47-53. (in Chinese)

[27] Q L J. Study on optimizing liquid pesticide application technology. Doctoral dissertation. Beijing: China Agricultural University, 2000. (in Chinese)

[28] Yuan H J, Wang G B. Effects of droplet size and deposition density on field efficacy of pesticides. Plant Protection, 2015; 41(6): 9-16. (in Chinese)

[29] Wang S L, Song J L, He X K, Li Y J, Ling Y. Design of air-assisted electric knapsack sprayer and experiment of its operation performance. Transactions of the CSAE, 2016; 32(21): 67-73. (in Chinese) 